\title{
Up-to-Date Role of CT/MRI LI-RADS in Hepatocellular Carcinoma
}

\author{
Guilherme Moura Cunha (iD) \\ Victoria Chernyak ${ }^{2}$ \\ Kathryn J Fowler' \\ Claude B Sirlin $\mathbb{D}^{\prime}$ \\ 'Liver Imaging Group, Department of \\ Radiology, University of California San \\ Diego, La Jolla, CA, USA; ${ }^{2}$ Department of \\ Radiology, Beth Israel Deaconess Medical \\ Center, Boston, MA, USA
}

\begin{abstract}
Hepatocellular carcinoma (HCC) is a leading cause of mortality worldwide and a major healthcare burden in most societies. Computed tomography (CT) and magnetic resonance imaging (MRI) play a pivotal role in the medical care of patients with or at risk for hepatocellular carcinoma (HCC). When stringent imaging criteria are fulfilled, CT and MRI allow for diagnosis, staging, and assessment of response to treatment, without the need for invasive workup, and can inform clinical decision making. Owing to the central role of these imaging modalities in HCC management, standardization is essential to facilitate proper imaging technique, accurate interpretation, and clear communication among all stakeholders in both the clinical practice and research settings. The Liver Imaging Reporting and Data System (LI-RADS) is a comprehensive system that provides standardization across the continuum of HCC imaging, including ordinal probabilistic approach for reporting that directs individualized management. This review discusses the up-to-date role of CT and MRI in HCC imaging from the LI-RADS perspective. It also provides a glimpse into the future by discussing how advances in knowledge and technology are likely to enrich the LI-RADS approach.
\end{abstract}

Keywords: hepatocellular carcinoma, computed tomography, magnetic resonance imaging, LI-RADS

\section{Introduction}

Hepatocellular carcinoma (HCC) is the fourth leading cause of cancer-associated mortality and a major healthcare burden worldwide. ${ }^{1}$ Imaging plays a pivotal role in the medical care of patients with or at risk for HCC. Accurate and reliable noninvasive diagnosis of $\mathrm{HCC}$ can be made with computed tomography (CT) or magnetic resonance imaging (MRI) when stringent criteria are applied. ${ }^{2}$ Tumor staging and management planning are based largely on imaging findings, often without the need for further invasive workup. CT and MRI also provide an assessment of treatment response in patients with $\mathrm{HCC}$, which informs therapeutic decisions.

With the growing primacy of CT and MRI in the care of patients with HCC, standardization of imaging technique and interpretation is essential to allow accurate diagnosis and facilitate communication among stakeholders in clinical practice and the research setting. The Liver Imaging Reporting and Data System (LI-RADS) is a comprehensive system that standardizes all aspects of HCC imaging, including terminology, technique, interpretation, and reporting. LI-RADS is supported by the American College of Radiology (ACR) and by the American Association for the Study of Liver Disease (AASLD), and is becoming the most widely accepted and
Correspondence: Guilherme Moura Cunha Liver Imaging Group, Department of Radiology, University of California San Diego, 9500 Gilman Dr. Mail Code 0888, La Jolla, San Diego, CA, 92093, USA

Email gcunha@uw.edu 
sophisticated diagnostic system for $\mathrm{HCC}$ in North America. In 2020, the AASLD recognized the utilization of CT/MRI LI-RADS as a metric of high-quality clinical care in patients with or at risk for HCC. It is anticipated that this recognition will incentivize the clinical adoption of LI-RADS by centers across the USA and may stimulate the utilization of LI-RADS in other countries as well.

This review focuses on the established role of CT/MRI LI-RADS in HCC diagnosis, staging, and assessment of treatment response. We also discuss emerging data on the role of MRI in HCC screening/surveillance and in the assessment of tumor biology and prognostication. Finally, we briefly introduce and discuss recent advances in imaging technology and computer science (eg, artificial intelligence) that may expand the role of imaging in the care of patients with or at risk for HCC.

\section{Overview of CT/MRI LI-RADS Algorithms and Key Concepts LI-RADS Algorithms}

For CT and MRI, LI-RADS provides two algorithms addressing two clinical contexts: CT/MRI LI-RADS, for HCC diagnosis and staging, and the LI-RADS treatment response algorithm (LR-TRA), for assessment of response to locoregional treatment.

The CT/MRI LI-RADS diagnostic algorithm is designed to ensure high specificity and positive predictive value (PPV) for the diagnosis of $\mathrm{HCC}$, and to this end should only be applied in populations with a high pretest probability of HCC, ie, the "high-risk population". This "high-risk population" includes adult patients with liver cirrhosis, patients with chronic hepatitis B viral infection with or without cirrhosis, and patients with current or prior HCC. In these populations, the expected PPV of LI-RADS for HCC diagnosis is $>95 \%{ }^{3}$ Although emerging data suggest that the performance of LI-RADS for the diagnosis of HCC may be equally high in the context of other risk factors for HCC, such as some patients with nonalcoholic fatty liver disease or chronic hepatitis $\mathrm{C}$ without cirrhosis, ${ }^{4}$ additional scientific evidence is needed to support the application of LI-RADS in these populations, ideally from large prospective clinical studies. Hence, to date, LIRADS should not be applied when the risk for HCC is low or not well established. This includes pediatric patients, those with other chronic liver diseases in the absence of cirrhosis, or in conditions where the PPV is reduced owing to the presence of nodules that mimic HCC on imaging, such as in patients with congenital hepatic fibrosis or patients with vascular causes of cirrhosis.

Similarly, the CT/MRI - LI-RADS treatment response algorithm (LR-TRA) is designed to detect and report residual viable HCC after locoregional therapy or resection, in the same high-risk population described above. Locoregional therapies include ablation therapies (radiofrequency, microwave, cryoablation, and ethanol injection), transcatheter therapies (conventional and drug-eluting bead chemoembolization, bland embolization, and radioembolization), and external beam radiation therapy. ${ }^{5}$ Emerging data suggest that the LR-TRA provides high PPV for viable tumors after treatment, ${ }^{6,7}$ although further data are to fully understand how it may be used to guide transplant eligibility or the need for retreatment. One caveat, though, is that the algorithm is not designed for assessing the response to systemic therapy, which is often at the patient-level rather than a lesion-level assessment. Readers should refer to other systems, such as modified RECIST, for this context of use. ${ }^{8}$

\section{LI-RADS Imaging Technical Specifications}

$\mathrm{CT}$ and MRI examinations need to meet minimal technical requirements to yield the desired diagnostic accuracy. The use of multidetector CT ( $\geq 8$ detectors) or 1.5 or 3 Tesla MR magnets is required, and the administration of intravenous contrast and multi-phase imaging are mandatory for HCC diagnosis and treatment response assessment. MRI has been shown to have higher sensitivity for the diagnosis of HCC than CT, particularly with the use of hepatobiliary contrast agents (HBAs), ie, gadoxetate disodium or gadobenate dimeglumine. ${ }^{9,10}$ However, LIRADS does not recommend one modality over another and does not advocate a specific type of MRI contrast agent. The selection of modality and contrast often depends on patient factors, institutional preferences, and other considerations.

With regard to the imaging study, the most important aspect is the acquisition of a technically adequate and welltimed arterial phase, as this phase is used to characterize the presence of arterial phase hyperenhancement (APHE), a hallmark and required feature for diagnosing $\mathrm{HCC}$ on imaging. Arterial phase imaging is subdivided into the early and the late arterial phases. LI-RADS strongly recommends that imaging be performed during the late arterial phase, ie, when the hepatic artery and portal vein are both enhanced, as this corresponds to the time when arterialized tumor tissue is maximally enhanced relative to background liver. If images are acquired too early, there may be little or 
no enhancement of arterialized tumor tissue, making the detection of APHE unreliable. For further discussion of arterial phase technical acquisition, the interested reader is referred to the LI-RADS v2018 Manual, Chapter 12, and other publications on this topic. ${ }^{11-14}$

Beyond the arterial phase, the liver protocol CT or MRI must also include a portal venous and delayed phase. The latter is acquired about 2-5 minutes after contrast injection and applies to CT and to MRI with extracellular agents. Readers should be aware that examinations missing any of these phases do not comply with LI-RADS requirements and may need to be repeated. When using gadoxetate disodium, a conventional delayed phase is not obtained; instead, images are acquired in the transitional and hepatobiliary phases at about 2-5 minutes and at about 15-20 minutes after injection, respectively. If gadobenate dimeglumine is used, a conventional delayed phase at about 2-5 minutes is usually acquired with optional acquisition of hepatobiliary phase images at around 1-3 hours after injection. Additional details on CT and MRI requirements can be found in the LI-RADS v2018 Manual. ${ }^{11}$

In addition to assessing the technical adequacy of an examination, ie, that all post-contrast phases are acquired, the interpretation adequacy should be determined. This refers to whether the study is significantly degraded by motion or other artifacts. LI-RADS currently does not provide guidance on scoring the severity of artifact; however, radiologists may choose to assign an LR-NC (LI-RADS Not Categorizable) category when an observation cannot be fully assessed owing to missing or degraded images, as described below, in "LIRADS Reporting". In these cases, the radiologist should use their judgment in recommending either repeat or alternative imaging to better characterize the observation.

\section{LI-RADS Observations}

In LI-RADS, the term observation refers to a focal area within the liver with an imaging appearance that is distinctive from the background. An observation may be a true lesion (an observation that represents a pathologic abnormality) or a pseudolesion (an observation that does not represent a pathologic abnormality). ${ }^{15}$ Observations treated by locoregional therapy are called treated observations.

\section{LI-RADS Categories}

The LI-RADS CT/MRI algorithms guide the radiologist through a stepwise process to assign a unique category for each observation. LI-RADS currently includes eight diagnostic categories ("LR-") for untreated observations and four treatment response assessment categories ("LR-TR-") for
Table I CT/MRI LI-RADS Diagnostic Categories for Untreated Observations and Treatment Response Assessment Categories for Treated Observations

\begin{tabular}{|c|c|c|}
\hline \multicolumn{2}{|c|}{ LI-RADS Categories } & \multirow{2}{*}{$\begin{array}{l}\text { Definition } \\
\text { Not categorizable (due to } \\
\text { image omission or } \\
\text { degradation) }\end{array}$} \\
\hline $\begin{array}{l}\text { Diagnostic } \\
\text { Algorithm }\end{array}$ & LR-NC & \\
\hline & LR-I & Definitely benign \\
\hline & LR-2 & Probably benign \\
\hline & LR-3 & $\begin{array}{l}\text { Intermediate probability of } \\
\text { malignancy }\end{array}$ \\
\hline & LR-4 & Probably HCC \\
\hline & LR-5 & Definitely HCC \\
\hline & LR-M & $\begin{array}{l}\text { Probably or definitely } \\
\text { malignant, not necessarily } \\
\text { HCC }\end{array}$ \\
\hline & LR-TIV & Tumor in vein \\
\hline \multirow[t]{4}{*}{$\begin{array}{l}\text { Treatment } \\
\text { Response } \\
\text { Algorithm }\end{array}$} & $\begin{array}{l}\text { LR-TR } \\
\text { Nonevaluable }\end{array}$ & $\begin{array}{l}\text { Treated, response not } \\
\text { evaluable (due to image } \\
\text { omission or degradation) }\end{array}$ \\
\hline & $\begin{array}{l}\text { LR-TR } \\
\text { Nonviable }\end{array}$ & $\begin{array}{l}\text { Treated, probably or definitely } \\
\text { not viable }\end{array}$ \\
\hline & $\begin{array}{l}\text { LR-TR } \\
\text { Equivocal }\end{array}$ & Treated, equivocally viable \\
\hline & LR-TR Viable & $\begin{array}{l}\text { Treated, probably or definitely } \\
\text { viable }\end{array}$ \\
\hline
\end{tabular}

treated observations (Table 1). Figure 1 illustrates the LIRADS diagnostic categories for untreated observations in the flow of the diagnostic algorithm. The diagnostic categories reflect the relative probability of an observation being benign, HCC, a malignancy other than HCC, or a malignancy associated with tumor in vein (TIV). ${ }^{16}$ The treatment response categories reflect the relative probability of recurrent or residual viable tumor after locoregional therapy. When image degradation or omission precludes differentiation between likely benign categories (LR-1 and LR-2) and likely malignant categories (LR-4, LR-5, LR-M), untreated observations are categorized as LR-NC, and treated observations as nonevaluable (LR-TR Nonevaluable).

\section{LI-RADS Reporting}

LI-RADS provides guidance on radiology reporting to ensure the inclusion of all relevant information. ${ }^{17}$ To 


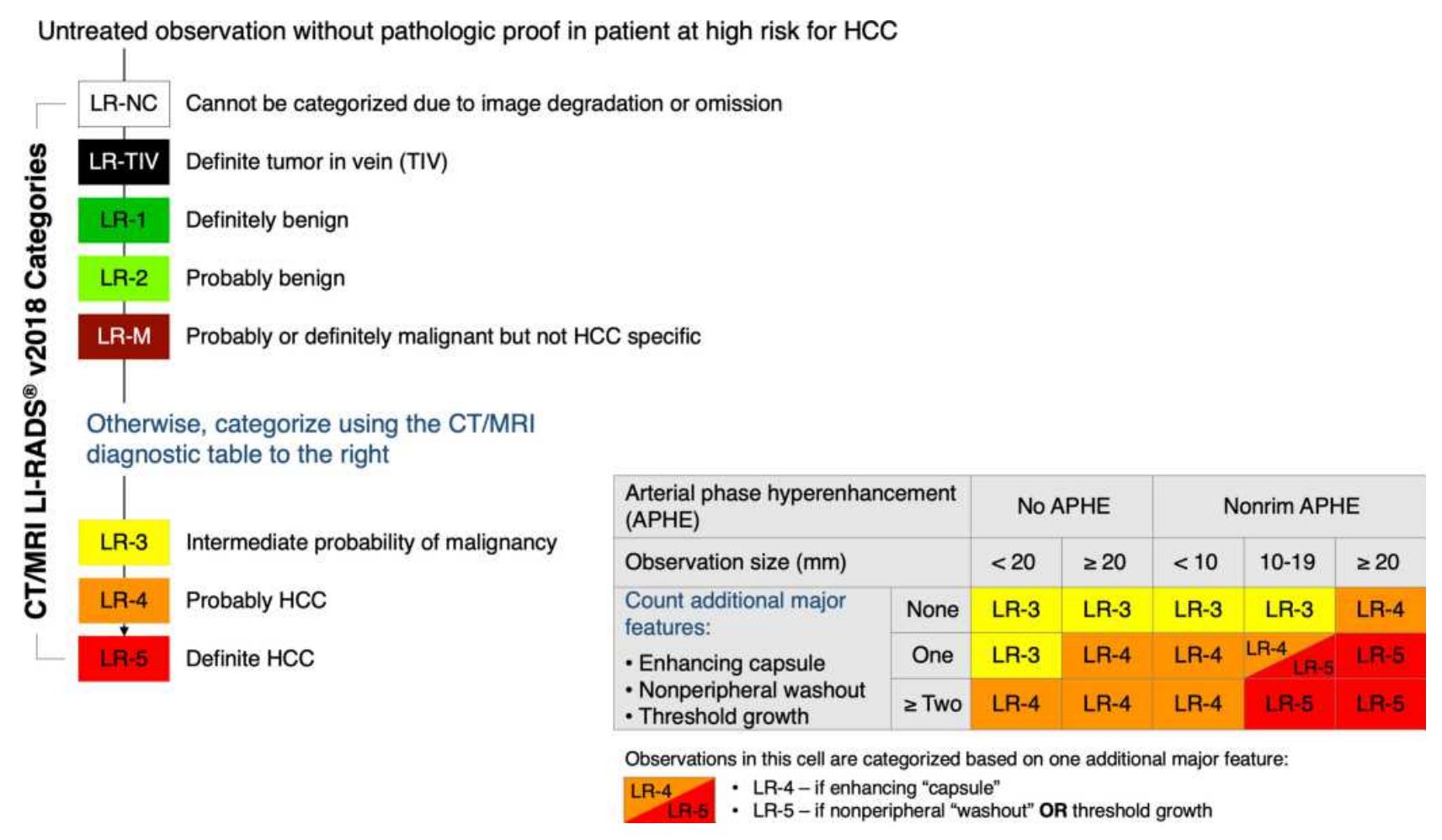

Figure I LI-RADS categories within the LI-RADS diagnostic algorithm.

Note: Adapted with permission from ACR Press. American College of Radiology. CT/MRI LI-RADS $®$ v2018.16. ${ }^{16}$

facilitate compliance, approved sample reporting templates are available free of charge in the LI-RADS Manual, Chapter $14,{ }^{11}$ for use by radiologists and other specialists. Observations categorized as LR-1 (definitely benign) or LR-2 (probably benign) can be reported at the radiologist's discretion. Observations categorized as LR-3 (intermediate probability of malignancy) can be reported either individually or in aggregate, depending on the number and the presence of observations with higher categories elsewhere in the liver. Observations categorized as LR-4 (probably HCC), LR-5 (definitely HCC), LR-M (probably or definitely malignant, not specific for HCC), LR-TIV (tumor in vein), and LR-NC, as well as treated observations, are reported individually. When multiple observations are present, radiologists may report them in aggregate, or as a combination of individual and aggregate reporting, selecting the option that most clearly and effectively communicates the findings. ${ }^{16}$ To facilitate more clear communication, radiologists are encouraged to report the most likely etiologies for observations categorized as LR-TIV and LR-M. ${ }^{16}$ For observations categorized as LR-NC or LR-TR Nonevaluable, radiologists are encouraged to indicate the cause of this categorization (ie, severe image degradation or image omissions), and to recommend repeat or alternative imaging as appropriate.

\section{The Role and Value of CT/MRI LI-RADS}

The main roles of CT and MRI in the medical care of HCC are diagnosis, preoperative staging, and detection of residual or recurrent disease after locoregional therapies. Both modalities have high enough specificity so that once stringent imaging criteria for $\mathrm{HCC}$ are fulfilled, treatment can be initiated without additional diagnostic workup (eg, biopsy). While both modalities have been investigated for HCC surveillance, with higher accuracy than ultrasound and/or serum biomarkers, ${ }^{18-20} \mathrm{CT}$ and MRI are not currently recommended by national clinical practice guidelines for surveillance owing to their presumed lower cost-effectiveness. ${ }^{21}$ As technology advances and data accumulate, it is likely that the role of these two modalities will expand to cover additional contexts in the care of patients with or at risk for HCC. In the next sections, we discuss the established and potential future roles or CT and MRI in HCC care through the lens of LI-RADS. 


\section{Role in HCC Diagnosis}

The CT/MRI LI-RADS diagnostic algorithm includes a stepwise decision tree and diagnostic table to steer the radiologist in assigning the most appropriate category. The first consideration in the algorithm is LR-NC, assigned when image omission or degradation precludes adequate evaluation of an observation, as described above. The next category is LR-TIV, assigned when there is definite enhancing tissue with a vein, indicating the presence of tumor in vein. Once thought to be exclusively seen in association with HCC, a recent meta-analysis found that nearly $30 \%$ of LRTIV cases were due to non-HCC malignancies. ${ }^{22}$ When assigning the TIV category, radiologists are encouraged to describe the most likely etiology, $\mathrm{HCC}$ or possible non-HCC malignancy.

The next two categories (LR-1 and LR-2) are applied to observations that are either definitely benign or probably benign on the basis of the radiologist's existing knowledge. After excluding benign entities and LR-TIV, the next consideration is the LR-M category. The LR-M category aims to preserve the high specificity of the LR-5 category for HCC diagnosis while maintaining high sensitivity for malignancies other than HCC. ${ }^{23}$ LR-M observations exhibit imaging features of malignancy but not features that are specific for
HCC (Figure 2). These include, but are not limited to, targetoid morphology (eg, rim arterial phase hyperenhancement, peripheral washout, delayed central enhancement), or nontargetoid LR-M features, such as an infiltrative appearance, marked restriction on diffusion weighted images, ischemia, or necrosis. In the LR-M category, $93-99 \%$ of observations are malignant, with $22-36 \%$ being HCC. ${ }^{24,25}$ The most common differential diagnoses in the LR-M category are intrahepatic cholangiocarcinomas (iCCA), combined tumors (cHCC-CCA), and HCC with atypical features. As with LR-TIV, radiologists are encouraged to report the most likely etiology of LR-M observations when possible, noting that approximately $25-30 \%$ of LR-M observations are in fact atypical HCC.

If the above categories are considered and excluded, the radiologist is next steered to use the LI-RADS diagnostic table, which considers the probability of HCC for solid hepatocellular nodules or masses. The three categories in the diagnostic table are intermediate probability of malignancy (LR-3), probably HCC (LR-4), and definite HCC (LR-5). These categories are assigned based on combinations of major imaging features (ie, features that in combination result in the diagnosis of HCC). Major features include size, nonrim APHE, nonperipheral
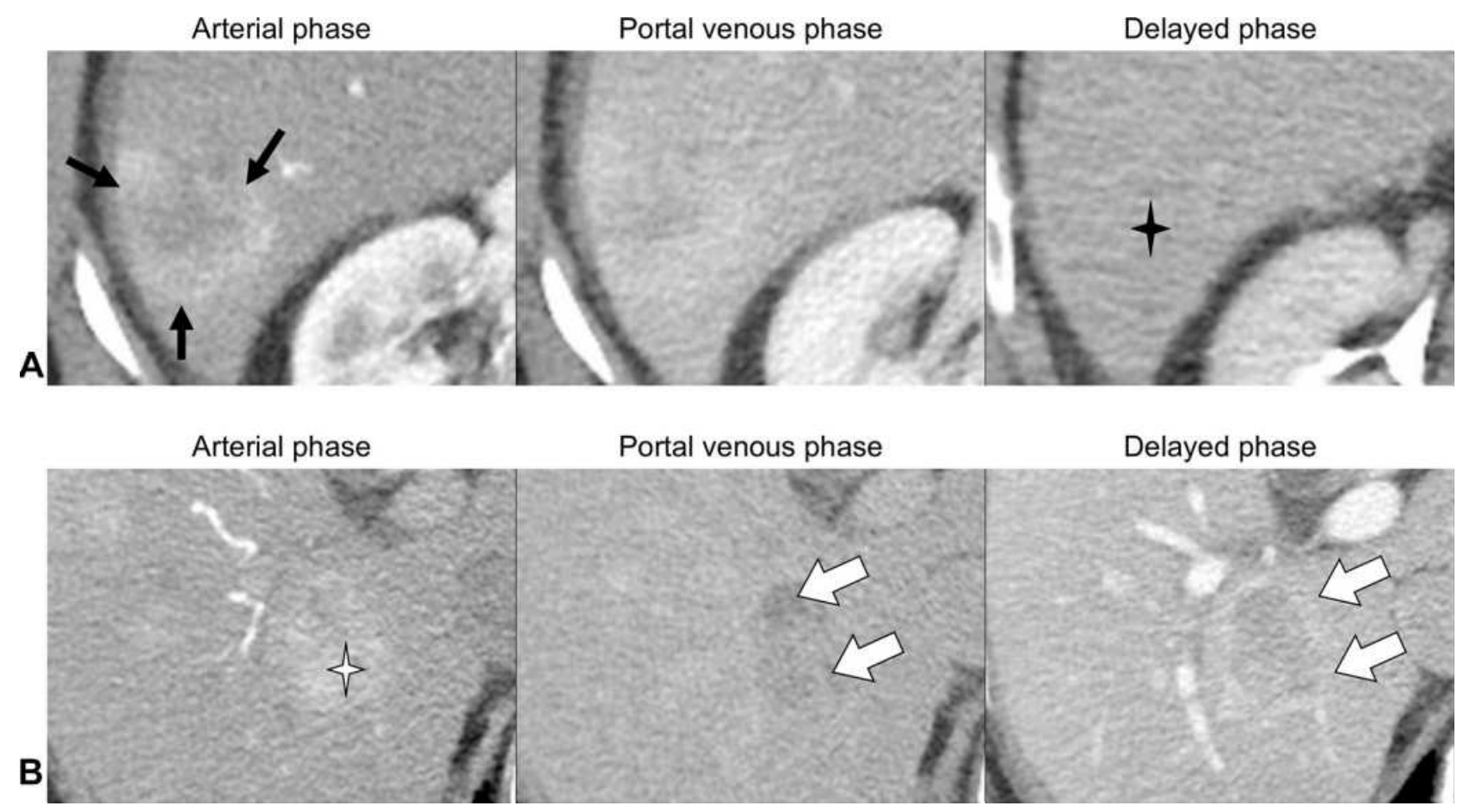

Figure 2 Contrast-enhanced CT images.

Notes: (A) LR-M observation measuring $3.9 \mathrm{~cm}$ in segment $\mathrm{VI}$ showing targetoid morphology; rim arterial phase hyperenhancement (black arrows) and delayed central enhancement (black star). (B) LR-5 observation (definite HCC) measuring $3.5 \mathrm{~cm}$ in segment 6 showing APHE (white star), washout and capsule (white arrows). 
washout, enhancing capsule, and threshold growth. The diagnostic table is constructed so that LR-3, LR-4, and LR-5 are assigned based on successively more stringent criteria. LI-RADS also provides numerous ancillary features, which can be used at the radiologist's discretion to facilitate detection of observations, increase diagnostic confidence, or adjust the final category. ${ }^{26}$ The major features, ancillary features, and LR-M features are discussed in more depth in the LI-RADS Manual and precisely defined in the LI-RADS lexicon. ${ }^{11,15}$

The prevalence of $\mathrm{HCC}$ and overall malignancies increases with the numerical hierarchical categories in the diagnostic table. In two meta-analyses, the prevalences of $\mathrm{HCC}$ and overall malignancy, respectively, were $38-40 \%$ and $34-40 \%$ for LR-3, $67-74 \%$ and 80-81\% for LR-4, and 92-94\%, and 97-99\% for LR$5 .^{23,24}$ The similarity in proportions between metaanalyses illustrate the overall robustness of the algorithm over time, since the two meta-analyses have a predominance of studies using different versions of the LI-RADS algorithm (v2017 vs v2014). ${ }^{24,25}$ This balance between the high specificity and PPV of the LR-5 category for the diagnosis of $\mathrm{HCC}$ and the high sensitivity of the LR-M category for malignancies in general is particularly important when guiding management decisions. While LR-M observations usually undergo additional diagnostic workup, often involving biopsy, LR-5 indicates confirmed HCC, prompting tumor staging and treatment planning without routine histologic confirmation. Table 2 summarizes the diagnostic performances of the LR-5 and LR-M categories on CT and MRI for the diagnosis of $\mathrm{HCC}$ and other malignancies, respectively.

\section{Role in HCC Staging}

For patients with an observation meeting LR-5 criteria, the next step in management is to evaluate disease burden (ie, tumor staging) and background liver features for treatment planning. Curative options for HCC include transplantation and resection. Resection opportunities are often limited in Western populations owing to advanced cirrhosis and limited functional liver reserve. Transplantation is curative for early-stage HCC and, by removing the field defect, reduces the risk of postoperative recurrence. In the USA, the United Network for Organ Sharing (UNOS) and the Organ Procurement and Transplantation Network (OPTN) are the national organizations that create policy and govern allocation and access to liver transplantation. There are more patients in need of a liver transplantation
Table 2 Sensitivity and Specificity of the LR-5 and LR-M Categories on CT and MRI for the Diagnosis of HCC and Other Malignancies, Respectively

\begin{tabular}{|c|c|c|c|c|}
\hline \multirow[t]{2}{*}{$\begin{array}{l}\text { LI-RADS } \\
\text { Category }\end{array}$} & \multirow[t]{2}{*}{$\begin{array}{l}\text { Imaging } \\
\text { Modality }\end{array}$} & \multicolumn{2}{|c|}{$\begin{array}{c}\text { Diagnostic } \\
\text { Performance }\end{array}$} & \multirow[t]{2}{*}{ Citation } \\
\hline & & Sensitivity & Specificity & \\
\hline \multirow[t]{4}{*}{ LR-5 } & MRI* & 0.69 & 0.98 & Cho, $2020^{23}$ \\
\hline & MRI† & 0.71 & 0.92 & Byun, $2020^{69}$ \\
\hline & CT/MRI* & 0.67 & 0.92 & Lee, 2020 \\
\hline & MRI & 0.80 & 0.90 & Ren $2019^{70}$ \\
\hline \multirow[t]{3}{*}{ LR-M } & MRI* & 0.89 & 0.88 & Cho, $2020^{23}$ \\
\hline & $\mathrm{CT}$ & 0.50 & 0.93 & An, $2019^{71}$ \\
\hline & MRI† & 0.57 & 0.94 & An, $2019^{71}$ \\
\hline
\end{tabular}

Notes: *Extracellular and hepatobiliary contrast agents. †Hepatobiliary contrast agent only. ‡Extracellular contrast agent only.

than there are livers available. Current UNOS/OPTN guidelines grant priority for patients with advanced liver dysfunction, ie, those who are most likely to die of their liver disease within the next 90 days, but also for patients with $\mathrm{T} 2$ stage $\mathrm{HCC}^{27}$ The rationale is that patients with early-stage HCC have markedly improved survival following transplantation but would not otherwise have access to transplantation on the basis of their liver function. The UNOS/OPTN HCC stage is determined primarily by CT or MRI, with biopsy typically reserved for observations not meeting strict imaging criteria for HCC. T2 stage HCC is defined as one $\mathrm{HCC} \geq 2 \mathrm{~cm}$ and $\leq 5 \mathrm{~cm}$ or up to $3 \mathrm{HCCs}$, each $\leq 3 \mathrm{~cm}$ on imaging. ${ }^{28}$ Figure 3 illustrates the UNOS/ OPTN radiologic staging system for HCC. Patients who present with T2 stage HCC may be listed for transplantation and receive priority on the waiting list, potentially bypassing others on the list.

In 2013, OPTN issued formal imaging criteria for the diagnosis of HCC. ${ }^{29}$ Only observations categorized as definite HCC on imaging according to OPTN criteria or histology-proven HCCs contribute to staging. Currently, the imaging criteria for definite HCC on CT and MRI between LI-RADS (LR-5) and UNOS/OPTN (OPTN Class 5) are similar but not identical. Observations that are 10-19 $\mathrm{mm}$ in size, with nonrim APHE and nonperipheral washout, are categorized as LR-5, while such observations do not meet OPTN-5 criteria. For OPTN, observations $10-19 \mathrm{~mm}$ in size require nonrim APHE, nonperipheral washout, and an enhancing capsule, or 
T1

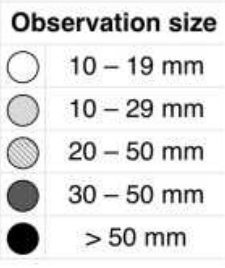

T2

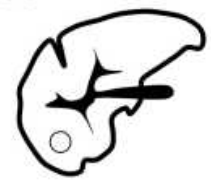

- > $>50 \mathrm{~mm}$
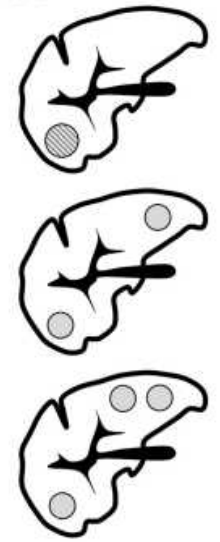

T3
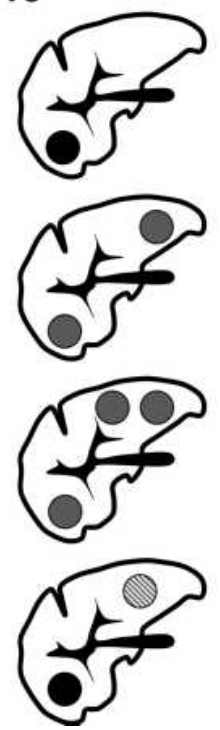

T4
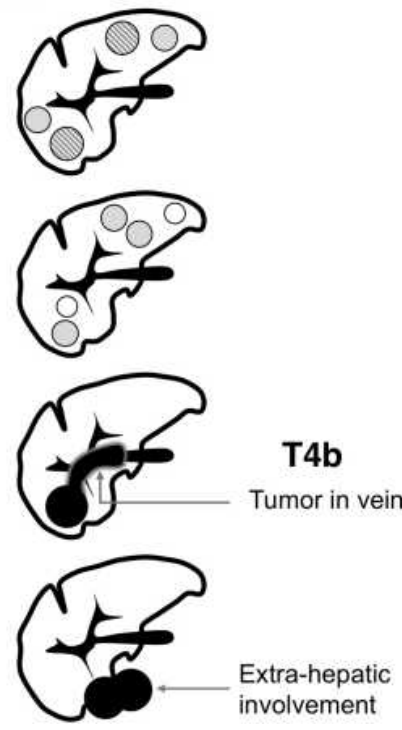

Figure 3 Schematic representation of the radiologic staging system for HCC adopted by UNOS/OPTN.

nonrim APHE and threshold growth, to meet the criteria for OPTN Class $5 .^{28}$ Therefore, not all LR-5 observations count toward radiologic staging for liver transplantation purposes in the USA. In addition, for staging purposes, OPTN uses qualifiers to describe observations within certain size thresholds or treated observations. OPTN Class $5 \mathrm{~A}$ and $\mathrm{B}$ describe observations $\geq 1 \mathrm{~cm}$ and $<2 \mathrm{~cm}$ and $\geq 2 \mathrm{~cm}$ and $\leq 5 \mathrm{~cm}$ in size, respectively. OPTN Class $5 \mathrm{X}$ indicates $\mathrm{HCC}$ beyond an acceptable size or stage for transplantation and OPTN Class 5T indicates HCC that has undergone locoregional therapies. ${ }^{29}$ LI-RADS does not use qualifiers to indicate size and has a separate algorithm to evaluate treated observations.

It bears mention that other LR categories, which have a high probability of being HCC, such as LR-4, do not contribute to OPTN staging. Likewise, OPTN has no equivalent to the LR-M category; however, these observations are often biopsied and treated on the basis of the pathologic diagnosis. Further research is needed to determine whether the presence of multiple LR-4 observations indicates a high likelihood of multifocal HCC and should be integrated into radiologic staging.

Given the mandatory 6-month wait imposed in most recent OPTN guidelines, many patients are bridged to transplantation with locoregional therapy. Hence, the treatment response algorithm is relevant for verifying successful downstaging and continued transplant eligibility following locoregional therapy. UNOS/OPTN does not provide specific guidance on assigning post-treatment stage, and transplant centers vary in how they incorporate treated lesions in their listing decisions. At the authors' institutions, any residual viable tumor in observations categorized as LRTR Viable contributes to staging and exception point extension. By comparison, treated observations categorized as LR-TR Equivocal or LR-TR Nonviable are not considered.

It is hoped and anticipated that OPTN will eventually adopt LR-5 as the radiologic definition of HCC, thereby achieving unification with LI-RADS and AASLD. Currently, OPTN provides only a binary classification, ie, HCC vs not HCC, with the aim of determining transplantation eligibility. Conversely, LI-RADS offers more granular, probability-based categories that allow for tailored management recommendations. While the relevance of these other categories in determining transplant eligibility is not yet established, LR-5 provides sufficiently high specificity for HCC to justify its adoption by OPTN.

\section{Role in Management Determinations}

In addition to providing a stage and helping to direct therapy for HCC, LI-RADS offers management recommendations for indeterminate findings, including imaging follow-up with an alternative imaging modality or additional diagnostic workup. ${ }^{30}$ In 2018, LI-RADS was incorporated into the AASLD HCC practice guidance, ${ }^{21}$ which describes standardized management suggestions linked to the individual diagnostic categories on CT or MRI.

For LR-5 observations, staging and treatment planning are usually recommended, without the need for additional 
workup. ${ }^{21}$ Histologic assessment may be pursued in some specific contexts, such as for systemic therapy or in clinical trials where molecular profiling may be necessary. ${ }^{31}$ For LR4 observations, management options may include biopsy, if the histologic diagnosis may impact clinical decisions or, alternatively, short interval imaging follow-up (usually in $\leq 3$ months), if supported by multidisciplinary discussion. In some cases, and in the context of multidisciplinary discussion, LR-4 observations can be treated without histologic confirmation. Biopsy is typically recommended for LR-M observations owing to the high probability of malignancies other than HCC, unless resection is a primary consideration; ${ }^{21}$ in that situation, resection can be performed without biopsy if the surgical approach would not be altered by the histologic diagnosis. LR-3 observations have intermediate risk of malignancy, and therefore, short-term imaging follow-up usually suffices, although biopsy may be pursued if recommended by multidisciplinary discussion. In patients with LR-1 and LR-2 observations, and patients with no focal observations, the return to routine surveillance is usually recommended. Management of LR-NC and LRTIV observations, as well as a summary of recommendations tailored to LI-RADS categories, are presented in Figure 4.

It is important to understand that management decisions do not flow solely from LI-RADS categories but must also incorporate patient-specific information - such as patient preferences, general health and comorbidities, treatment history, social support system, and health insurance - frequently unknown to the radiologist. ${ }^{32}$ Levels of circulating biomarkers such as alpha-fetoprotein (AFP) also may impact the final management decision.
Similarly, management may be influenced by institutional or regional practice patterns and resources. ${ }^{33}$

\section{Role in Assessing Response to Treatment}

Several treatment options are available for patients with $\mathrm{HCC}$, including locoregional therapies, surgical resection, transplantation, and systemic therapies. These can have different objectives, such as cure, downstaging, bridging, debulking, or palliation. CT and MRI play an essential role in assessing response to therapy and determining further management after treatment. This is particularly true for downstaging and bridging options, which aim to reduce the risk of disease progression and waiting list dropout. ${ }^{28}$

While other HCC diagnostic algorithms provide little or no guidance for assessing response to treatment, LI-RADS has developed a treatment response algorithm (LR-TRA) to assess and report response to locoregional therapies. The LR-TRA comprises four categories to assess response in individual treated observations on CT or MRI, ie, a lesionlevel response assessment. The LR-TRA categories are LRTR Nonevaluable, LR-TR Nonviable, LT-TR Equivocal, and LR-TR Viable. Similarly to LR-NC in the diagnostic algorithm, LR-TR Nonevaluable is reserved for cases where image omission or degradation precludes the evaluation of treatment response. Treated observations showing a complete lack of contrast enhancement on CT or MRI are categorized as TR-Nonviable. Observations that exhibit a nodular or mass-like area of APHE, washout, or enhancement similar to pre-treatment on post-treatment imaging are considered to represent residual viable tumor and are assigned an LR-TR Viable category. When an LR-TR Viable observation is identified, radiologists are encouraged

\section{Multiphase CT or MRI}

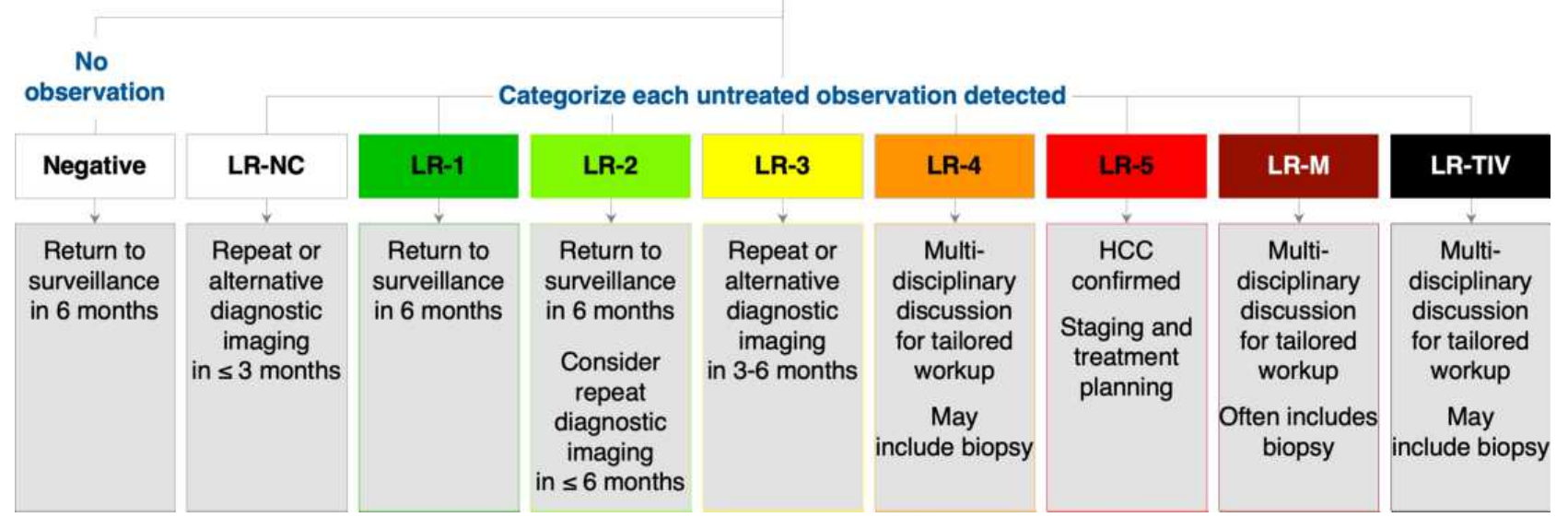

Figure 4 Management recommendations tailored to LI-RADS diagnostic categories.

Note: Adapted with permission from ACR Press. American College of Radiology. CT/MRI LI-RADS $®$ v2018.16. ${ }^{16}$ 

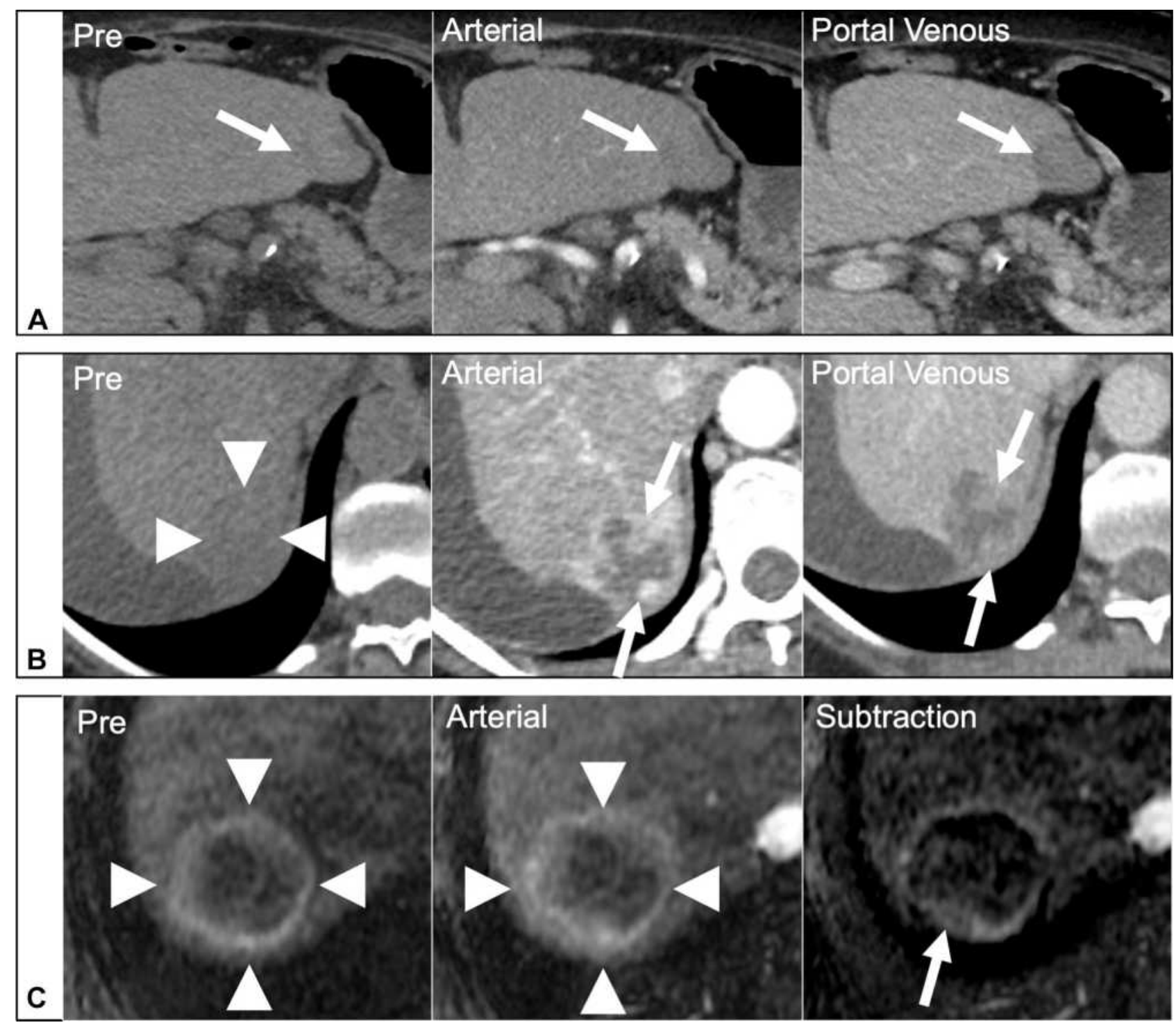

Figure 5 Assessment of response to locoregional therapies with the LI-RADS treatment response algorithm.

Notes: (A) Dynamic CT images showing a treated observation in segment 3 with complete lack of contrast enhancement (arrows), consistent with an LR-TR Nonviable observation. (B) Dynamic CT images showing a treated observation in segment 6 (arrowheads) with nodular areas of APHE and washout (arrows) consistent with an LR-TR Viable observation. (C) Pre-contrast and arterial phase MR images with image subtraction. A hyperintense rim is observed in a treated observation (arrowheads). On the subtraction image there is a thin rim of enhancement with a questionable posterior focal thickening (arrow), making difficult the differentiation between post-treatment changes and residual disease. An LR-TR Equivocal category was assigned.

to also report the pre-treatment LI-RADS category and size, as well as the longest dimension of the residual enhancing component. This efficiently communicates the level of response and informs the need for retreatment or alternative treatments. The LR-TR Equivocal category aims to communicate uncertainty in differentiating viable tumors from imaging features associated with post-treatment changes. Figure 5 shows examples of lesions categorized as LR-TR Viable, LR-TR Nonviable, and LR-TR Equivocal.

Although the LR-TRA is less well validated than the diagnostic algorithm, evidence supporting its use is emerging. Studies have shown that the LR-TR Viable category conveys high specificity and PPV for the detection of residual tumor (Table 3 ). Nevertheless, the interpretation of liver imaging in the post-treatment setting can be difficult owing to the wide range of available treatments with potentially different mechanisms of action and imaging appearances. The challenge is compounded by variability in the time interval between imaging and therapy, progressive parenchymal damage from incremental treatments, and variable degrees of liver decompensation. ${ }^{5}$ The LR-TR Equivocal category communicates uncertainty and 
Table 3 Specificity and PPV of the LR-TR Viable Category for the Diagnosis of Residual Tumor

\begin{tabular}{|c|c|c|c|c|c|c|c|c|}
\hline \multirow{2}{*}{$\begin{array}{l}\text { LR-TR } \\
\text { Category }\end{array}$} & \multirow{2}{*}{$\begin{array}{l}\text { Imaging } \\
\text { Modality }\end{array}$} & \multirow{2}{*}{$\begin{array}{c}\text { Treatment } \\
\text { Modality }\end{array}$} & \multirow{2}{*}{$\begin{array}{c}\text { Inter- } \\
\text { Reader } \\
\text { Agreement } \\
\text { (k) }\end{array}$} & \multicolumn{2}{|c|}{ Performance } & \multirow{2}{*}{$\begin{array}{l}\text { Prevalence of } \\
\text { Residual } \\
\text { Viable Tumor } \\
\text { (\%) }\end{array}$} & \multirow{2}{*}{$\begin{array}{l}\text { Reference } \\
\text { Standard }\end{array}$} & \multirow[t]{2}{*}{ Citation } \\
\hline & & & & $\begin{array}{l}\text { Specificity for } \\
\text { Residual } \\
\text { Viable Disease } \\
\text { (\%) }\end{array}$ & $\begin{array}{c}\text { PPV for } \\
\text { Residual } \\
\text { Viable } \\
\text { Disease (\%) }\end{array}$ & & & \\
\hline \multirow[t]{7}{*}{ TR-Viable } & MRI & $\begin{array}{l}\text { RFA or } \\
\text { MWA }\end{array}$ & 0.71 & $85-97$ & - & 41 & Explant & $\begin{array}{c}\text { Chaudhry, } \\
2020^{34}\end{array}$ \\
\hline & MRI & $\begin{array}{l}\text { RFA or } \\
\text { MWA }\end{array}$ & 0.75 & 99 & 93 & 39 & Explant & $\begin{array}{l}\text { Cools, } \\
2020^{7}\end{array}$ \\
\hline & $\begin{array}{c}\mathrm{CT} \text { and } \\
\mathrm{MRI}\end{array}$ & TABE & 0.55 & - & $86-92$ & - & Explant & $\begin{array}{c}\text { Shropshire, } \\
2019^{6}\end{array}$ \\
\hline & $\mathrm{CT}$ & \multirow{2}{*}{$\begin{array}{c}\text { All } \\
\text { locoregional }\end{array}$} & - & 90 & - & - & \multirow{2}{*}{$\begin{array}{l}\text { Resection } \\
\text { or explant }\end{array}$} & \multirow{2}{*}{$\begin{array}{l}\text { Park, } \\
2020^{72}\end{array}$} \\
\hline & MRI & & - & 83 & - & - & & \\
\hline & $\mathrm{CT}$ & \multirow{2}{*}{$\begin{array}{c}\text { All } \\
\text { locoregional }\end{array}$} & \multirow[t]{2}{*}{0.63} & 94 & _ & \multirow[t]{2}{*}{59} & \multirow[t]{2}{*}{ Explant } & \multirow{2}{*}{$\begin{array}{l}\text { Youn, } \\
2021^{73}\end{array}$} \\
\hline & MRI & & & 76 & - & & & \\
\hline
\end{tabular}

Abbreviations: RFA, radiofrequency ablation; MWA, microwave ablation; TABE, transarterial bead embolization.

may highlight these challenges, which may prompt additional imaging workup or retreatment. Two studies have shown a high frequency of histologically viable tumor in treated observations categorized as LR-TR Equivocal and sampled pathologically after subsequent explantation. ${ }^{6,34}$ The relevance of viable tumor in such cases is unclear, as it often represents viability on the microscopic scale. A separate study showed no significant difference in overall survival between patients with LR-TR Nonviable and LR-TR Equivocal observations. ${ }^{35}$ Further research is needed to elucidate the clinical relevance and optimal management of treated observations with equivocal viability on imaging.

The emerging scientific evidence will also help to refine the current algorithm to incorporate a better understating of its performance in relation to specific treatment modalities. Currently, the LR-TRA is intended for application in patients after locoregional therapy for HCC, ie, locoablative therapies, transcatheter therapies, and radiation therapies. ${ }^{4}$ In the context of radiation therapies (eg, transarterial Y-90 radioembolization, stereotactic body radiation therapy [SBRT]), the assessment of response on CT and MRI is particularly challenging and an area of active research. King et al reported lower inter-reader agreement for LR-TR categories and a higher frequency of TR-Equivocal categorization on early (4-6 weeks) versus late $(6-9$ months $)$ post-treatment imaging in patients undergoing radioembolization with Y-90. ${ }^{36}$ It is not uncommon for observations treated with these modalities to manifest a transient increase in size and/or in enhancement on imaging performed in the first 3-6 months, which may be misinterpreted as viable residual tumor. ${ }^{37}$ Although the LR-TRA may be applied to observations treated with radiation-based therapies, this should be done with caution. For these reasons, it is anticipated that the LI-RADS treatment response algorithm will be refined to better address response assessment after radiation-based therapies. A system for assessing response after systemic therapy is in the planning phases.

For patients treated with surgery, imaging is performed to ensure the completeness of tumor resection. In such cases, the LR-TRA may be applied to communicate surgical response and for determining management when observations suspicious for local recurrence/residual disease are seen along or contiguous with the surgical margin. However, new observations arising in the liver away from the resection bed should be categorized using the LIRADS CT/MRI diagnostic algorithm.

Unfortunately, the majority of patients with HCC do not qualify for curative therapies. For these patients, systemic options may be offered, and often include conventional chemotherapy, targeted therapy, and immunotherapy, either alone or in combination. Currently, the LR-TRA does not address systemic 
therapies and imaging studies of patients treated with systemic therapies are often evaluated using the modified Response Evaluation Criteria in Solid Tumors (mRECIST). In contrast to the LR-TRA, mRECIST is designed to provide a per-patient assessment of response instead of a per-lesion assessment. ${ }^{8,38}$

\section{Future Directions}

Beyond the most recent update of the diagnostic algorithm in 2018 and the incorporation of CT/MRI LI-RADS into the AASLD clinical guidance, ${ }^{39}$ LI-RADS is being actively refined and its applications are being expanded as evidence emerges and in response to users' feedback and new technologies. Some of the short-term future directions of LI-RADS are described below.

\section{Role and Value in HCC Surveillance}

Imaging surveillance reduces mortality in patients at risk for HCC as it allows tumor detection and treatment at earlier stages. ${ }^{40}$ Currently, HCC surveillance in at-risk populations is done using ultrasound every 6 months, relying on its wide availability and lower costs, ${ }^{21}$ and LIRADS has developed an ultrasound surveillance algorithm to guide proper sonographic technique and reporting of results. ${ }^{41}$ Ultrasound has limited sensitivity for small liver nodules, however, particularly in patients with liver cirrhosis and associated conditions such as obesity and fatty liver. A 2018 meta-analysis showed that the sensitivity of ultrasound for detecting early $\mathrm{HCC}$ can be as low as $47 \%{ }^{18}$ Reasons for the low sensitivity of ultrasound include parenchymal heterogeneity due to cirrhosis, which can obscure small nodules, shadowing from ribs, and attenuation of the ultrasound beam by overlying adiposity and/or by liver fat.

CT and MRI with extracellular contrast agents are less affected by these factors and so have significantly higher sensitivity than ultrasound for HCC screening in adults with cirrhosis. ${ }^{18,42}$ HBA-enhanced MRI has even better accuracy, with higher than $85 \%$ per-lesion sensitivity and 95\% PPV for HCC. ${ }^{10,42}$ However, the use of CT and MRI for HCC surveillance is impractical owing to their higher complexity and costs, and in the case of CT, uncertain risks associated with radiation exposure. To address some of these limitations, investigators have proposed abbreviated MRI (AMRI) protocols as an alternative approach for imaging-based HCC surveillance while avoiding the high costs and complexity associated with full diagnostic MRI protocols. ${ }^{43}$ These AMRI protocols are shorter than diagnostic studies, comprising only a small number of sequences that are tailored for screening or surveillance purposes, and can be performed in approximately $10 \mathrm{~min}$ utes of total MRI table time, significantly reducing patient burden and examination complexity when compared to full diagnostic studies. Three types of AMRI protocol have been proposed for HCC screening and surveillance: Non-contrast AMRI, Dynamic AMRI, and Hepatobiliary Phase AMRI. ${ }^{43}$ Each of these protocols has advantages and disadvantages. Although further research is needed to elucidate which is best, all have shown higher sensitivity than ultrasound for HCC detection. ${ }^{44}$ The higher sensitivity potentially could allow for longer surveillance intervals than the 6 months currently recommended with ultrasound, further reducing patient burden and costs. One study showed that among patients at risk for HCC who had a negative baseline gadoxetate acid-MRI, only $1 \%$ had clinically significant liver nodules after 1 year of followup. ${ }^{45}$ Despite these promising results, large prospective multicenter clinical trials are needed to determine the clinical value and optimal intervals of AMRI protocols for HCC surveillance before they can be incorporated widely into clinical practice.

LI-RADS does not currently provide an AMRI surveillance algorithm. As evidence accrues, it may be possible to perform a personalized surveillance strategy, with patients assigned to the particular modality (ultrasound, Non-contrast AMRI, Dynamic AMRI, Hepatobiliary Phase AMRI) most likely to be effective based on patient preferences, compliance, body habitus, severity of cirrhosis, degree of liver fat accumulation, and other factors.

\section{Role and Value in Assessing Biologic Behavior and Prognosis}

In many aspects, HCC is a heterogeneous neoplasm with different molecular profiles, and variable biology and behavior. HCCs with keratin 19 (K19) expression, for example, are associated with a more aggressive behavior, resistance to chemotherapy or locoregional therapy, and overall poorer prognosis than their counterparts. ${ }^{46}$ The ability to evaluate the tumor molecular profile and biology with diagnostic imaging studies may allow more individualized management of $\mathrm{HCC}$ in accordance with the modern concepts of precision medicine. A few studies have shown the ability of CT and MRI to assess molecular and biologic differentiation of HCCs. ${ }^{47-51}$ HBA-enhanced MRI seems to be particularly promising. HCCs that are 
hyperintense on hepatobiliary phase images have better prognosis, while HCCs with high tumor-to-liver signal ratio or with peritumoral hypointensity on hepatobiliary phase images are associated with worse molecular features, more aggressive behavior, and higher recurrence rates. ${ }^{50,52,53}$ Arterial phase imaging features may help to predict molecular differentiation and biologic behavior. In one study, the presence of irregular rim enhancement on arterial phase images was associated with worse histologic features, such as macrotrabecular pattern, immunomarkers of stemness, and microvascular invasion, and, therefore, worse outcomes. ${ }^{54}$

Other studies have shown that imaging features defined by LI-RADS as well as LI-RADS categories may stratify risk, predict response to treatment, and inform overall prognosis. ${ }^{47,51,53,55,56}$ In a study including HCCs, iCCA, and cHCC-CCA, the LI-RADS category was independently correlated with overall survival and recurrencefree survival. ${ }^{48}$ In that study, observations categorized as LR-4 or LR-5 had better outcomes than observations categorized as LR-M, regardless of their final histopathologic diagnosis. In another study investigating the application of LI-RADS for the diagnosis of cHCC-CCA, tumors exhibiting LI-RADS features of HCC (eg, nonrim APHE, capsule, nonperipheral washout) had significantly better outcomes with lower rates of post-surgical recurrence than tumors exhibiting LR-M features. ${ }^{55}$ In two separate studies, Wei et al found independent associations between LI-RADS major and ancillary features with tumor differentiation grade, presence of microvascular invasion, and recurrence rates, and suggested that some of these features could potentially be used as prognostic biomarkers in patients with HCC. ${ }^{51,53}$

\section{Structured Reporting}

The adoption of structured reports has been shown to improve clinical care by reducing the variability in key feature descriptions while promoting adherence to diagnostic systems. ${ }^{17,57}$ Initiatives to promote the adoption of LI-RADS structured reporting templates have been proposed by radiology societies in parallel with commercial interests to integrate LI-RADS algorithms into dictation and reporting applications. ${ }^{58,59}$ These mechanisms are likely to promote standardization across multiple institutions and clinical practices, facilitating the adoption of LIRADS. Such adoption will enable the development of geographically and practice-diverse registries of uniform curated data to facilitate research and enhance knowledge.

\section{New Technologies}

Advances in imaging technology have provided CT and MRI with the ability to accurately and non-invasively diagnose, grade, or stage the underlying liver disease that predisposes to HCC development. ${ }^{60,61}$ For example, fibrosis stage as well as liver fat have been shown to be independent risk factors for $\mathrm{HCC}$ development in patients with chronic hepatitis C. ${ }^{62}$ Hence, with the new diagnostic capabilities of CT and MRI, it is likely that these imaging modalities will have their roles expanded to predict the risk of HCC development, even when no focal observations are detected. Ichikawa et al demonstrated that the incidence of HCC was significantly different between patients with high $(>4.7 \mathrm{kPa})$, moderate $(3-4.7 \mathrm{kPa})$, or mild $(<3 \mathrm{kPa})$ liver stiffness, a biomarker of liver fibrosis, when measured with MR elastography during a 3-year follow-up period. ${ }^{63}$ Ajmera et al reported patients with higher amounts of liver fat, as measured by MRI-proton density fat fraction, to be at higher risk of progressing to advanced liver fibrosis, an established risk factor for HCC development. ${ }^{64}$ As evidence accumulates, these noninvasive imaging biomarkers may help to expand the population in which LI-RADS is applicable, and potentially be integrated into the algorithms to adjust or more accurately report the probability of HCC.

Artificial intelligence (AI) is being incorporated rapidly into radiology. Deep learning, a subtype of AI, has been extensively investigated as a means to increase the diagnostic performance of imaging studies owing to its remarkable ability to classify medical images. ${ }^{65}$ Naturally, a number of deep-learning-based algorithms have been applied to CT and MR images of patients at risk for HCC, for lesion detection, characterization, risk stratification, or prediction of response to treatment. Many of these studies relied on the standardization and rigor provided by LI-RADS for development, training, and validation of these AI algorithms. ${ }^{66-68}$ The combination of advances in imaging technology with developments in the computer science field lays out an exciting landscape for the near future, which is likely to widen the role of CT and MRI in the medical care of patients at risk for or with HCC.

\section{Conclusion}

$\mathrm{CT}$ and MRI yield high specificity for HCC diagnosis when stringent imaging criteria are met, obviating the need for invasive diagnostic workup. Tumor staging for treatment 
planning as well as assessment of response to treatment can also be accurately performed using these imaging modalities. As the role of these methods covers many components of the medical care of patients with $\mathrm{HCC}$, standardization is key to ensure proper technique, accurate interpretation, and unambiguous communication among healthcare professionals and researchers. The Liver Imaging Reporting and Data System (LI-RADS) standardizes all aspects of HCC imaging, permits probabilistic categorization, and facilitates personalized medical care. As the role of CT and MRI in the management of $\mathrm{HCC}$ expands to include screening and prognostication, it is anticipated that LI-RADS will be broadened to provide the required rigor and standardization for these additional imaging contexts.

\section{Disclosure}

Dr Guilherme Moura Cunha is a member of the ACR LIRADS steering committee; Dr. Sirlin reports grants from GE, Siemens, Philips, Bayer, Foundation of NIH, Gilead, and Pfizer (grant is to UW-Madison; UCSD is a subcontract to UW-Madison); personal consultation fees from Blade, Boehringer, and Epigenomics; consultation under the auspices of the University to AMRA, BMS, Exact Sciences, GE Digital, IBM-Watson, and Pfizer; lab service agreements from Enanta, Gilead, ICON, Intercept, Nusirt, Shire, Synageva, Takeda; royalties from Wolters Kluwer for educational material outside the submitted work; honoraria to the institution from Medscape for educational material outside the submitted work; ownership of stock options in Livivos; unpaid position in advisory board to Quantix Bio. The authors report no other conflicts of interest in this work.

\section{References}

1. Akinyemiju T, Abera S, Ahmed M, et al. The burden of primary liver cancer and underlying etiologies from 1990 to 2015 at the global, regional, and national level: results from the global burden of disease study 2015. JAMA Oncol. 2017;3(12):1683-1691. doi:10.1001/ jamaoncol.2017.3055

2. Kim TH, Kim SY, Tang A, Lee JM. Comparison of international guidelines for noninvasive diagnosis of hepatocellular carcinoma: 2018 update. Clin Mol Hepatol. 2019;25(3):245. doi:10.3350/ cmh.2018.0090

3. Chernyak V, Fowler KJ, Kamaya A, et al. Liver Imaging Reporting and Data System (LI-RADS) version 2018: imaging of hepatocellular carcinoma in at-risk patients. Radiology. 2018;289(3):816-830. doi:10.1148/radiol.2018181494

4. Ludwig DR, Fraum TJ, Cannella R, et al. Expanding the Liver Imaging Reporting and Data System (LI-RADS) v2018 diagnostic population: performance and reliability of LI-RADS for distinguishing hepatocellular carcinoma (HCC) from non-HCC primary liver carcinoma in patients who do not meet strict LI-RADS high-risk criteria. $H P B \quad$ (Oxford). 2019;21(12):1697-1706. doi:10.1016/j.hpb.2019. 04.007
5. Voizard N, Cerny M, Assad A, et al. Assessment of hepatocellular carcinoma treatment response with LI-RADS: a pictorial review. Insights Imaging. 2019;10(1):1-22. doi:10.1186/s13244-019-0801-z

6. Shropshire EL, Chaudhry M, Miller CM, et al. LI-RADS treatment response algorithm: performance and diagnostic accuracy. Radiology. 2019;292(1):226-234. doi:10.1148/radiol.2019182135

7. Cools KS, Moon AM, Burke LM, McGinty KA, Strassle PD, Gerber DA. Validation of the liver imaging reporting and data system treatment response criteria after thermal ablation for hepatocellular carcinoma. Liver Transpl. 2020;26(2):203-214. doi:10.1002/1t.25673

8. Yaghmai V, Besa C, Kim E, Gatlin JL, Siddiqui NA, Taouli B. Imaging assessment of hepatocellular carcinoma response to locoregional and systemic therapy. Am J Roentgenol. 2013;201(1):80-96. doi:10.2214/AJR.13.10706

9. Roberts LR, Sirlin CB, Zaiem F, et al. Imaging for the diagnosis of hepatocellular carcinoma: a systematic review and meta-analysis. Hepatology. 2018;67(1):401-421. doi:10.1002/hep.29487

10. Lee YJ, Lee JM, Lee JS, et al. Hepatocellular carcinoma: diagnostic performance of multidetector CT and MR imaging - a systematic review and meta-analysis. Radiology. 2015;275(1):97-109. doi:10.1148/radiol.14140690

11. Available from: https://www.acr.org/-/media/ACR/Files/ClinicalResources/LIRADS/LI-RADS-2018-Manual-5Dec18.pdf. Accessed January 14, 2021.

12. Hong SB, Lee NK, Kim S, et al. Modified CAIPIRINHA-VIBE without view-sharing on gadoxetic acid-enhanced multi-arterial phase MR imaging for diagnosing hepatocellular carcinoma: comparison with the CAIPIRINHA-Dixon-TWIST-VIBE. Eur Radiol. 2019;29(7):3574-3583. doi:10.1007/s00330-019-06095-x

13. Saranathan M, Rettmann DW, Hargreaves BA, Clarke SE, Vasanawala SS. Differential subsampling with cartesian ordering (DISCO): a high spatio-temporal resolution dixon imaging sequence for multiphasic contrast enhanced abdominal imaging. J Magn Reson Imaging. 2012;35(6):1484-1492. doi:10.1002/jmri.23602

14. Ikram NS, Yee J, Weinstein S, et al. Multiple arterial phase MRI of arterial hypervascular hepatic lesions: improved arterial phase capture and lesion enhancement. Abdom Radiol. 2017;42(3):870-876. doi:10.1007/s00261-016-0948-8

15. Available from: https://www.acr.org/-/media/ACR/Files/RADS/LIRADS/Lexicon-Table-2020.pdf. Accessed January 14, 2021.

16. Available from: https://www.acr.org/-/media/ACR/Files/RADS/LIRADS/LI-RADS-2018-Core.pdf. Accessed January 14, 2021.

17. Flusberg M, Ganeles J, Ekinci T, et al. Impact of a structured report template on the quality of CT and MRI reports for hepatocellular carcinoma diagnosis. J Am Coll Radiol. 2017;14(9):1206-1211. doi:10.1016/j.jacr.2017.02.050

18. Tzartzeva K, Obi J, Rich NE, et al. Surveillance imaging and alpha fetoprotein for early detection of hepatocellular carcinoma in patients with cirrhosis: a meta-analysis. Gastroenterology. 2018;154 (6):1706-1718. doi:10.1053/j.gastro.2018.01.064

19. Kim SY, An J, Lim YS, et al. MRI with liver-specific contrast for surveillance of patients with cirrhosis at high risk of hepatocellular carcinoma. JAMA Oncol. 2017;3(4):456-463. doi:10.1001/ jamaoncol.2016.3147

20. Yoon JH, Lee JM, Lee $\mathrm{DH}$, et al. A comparison of biannual two-phase low-dose liver CT and US for HCC surveillance in a group at high risk of HCC development. Liver Cancer. 2020;9 (5):503-517. doi:10.1159/000506834

21. Marrero JA, Kulik LM, Sirlin CB, et al. Diagnosis, staging, and management of hepatocellular carcinoma: 2018 practice guidance by the American Association for the Study of liver diseases. Hepatology. 2018;68(2):723-750. doi:10.1002/hep.29913

22. Kim DH, Choi SH, Park SH, et al. The liver imaging reporting and data system tumor-in-vein category: a systematic review and meta-analysis. Eur Radiol. 2020;1-10. 
23. Cho MJ, An C, Aljoqiman KS, et al. Diagnostic performance of liver imaging reporting and data system in patients at risk of both hepatocellular carcinoma and metastasis. Abdom Radiol. 2020;45 (11):3789-3799. doi:10.1007/s00261-020-02581-9

24. Lee S, Kim -S-S, Roh YH, Choi J-Y, Park M-S, Kim M-J. Diagnostic performance of CT/MRI liver imaging reporting and data system v2017 for hepatocellular carcinoma: a systematic review and meta-analysis. Liver Int. 2020;40(6):1488-1497. doi:10.1111/liv.14424

25. Van der Pol CB, Lim CS, Sirlin CB, et al. Accuracy of the liver imaging reporting and data system in computed tomography and magnetic resonance image analysis of hepatocellular carcinoma or overall malignancy - a systematic review. Gastroenterology. 2019;156(4):976-986. doi:10.1053/j.gastro.2018.11.020

26. Cerny M, Bergeron C, Billiard JS, et al. LI-RADS for MR imaging diagnosis of hepatocellular carcinoma: performance of major and ancillary features. Radiology. 2018;288(1):118-128. doi:10.1148/ radiol.2018171678

27. Available from: https://optn.transplant.hrsa.gov/governance/policies. Accessed January 14, 2021.

28. Cunha GM, Tamayo-Murillo DE, Fowler KJ. LI-RADS and transplantation: challenges and controversies. Abdom Radiol. 2019;6:1-4.

29. Wald C, Russo MW, Heimbach JK, Hussain HK, Pomfret EA, Bruix J. New OPTN/UNOS policy for liver transplant allocation: standardization of liver imaging, diagnosis, classification, and reporting of hepatocellular carcinoma. Radiology. 2013;266(2):376-382. doi:10.1148/radiol.12121698

30. Mitchell DG, Bashir MR, Sirlin CB. Management implications and outcomes of LI-RADS-2,-3,-4, and-M category observations. Abdom Radiol. 2018;43(1):143-148. doi:10.1007/s00261-017-1251-z

31. Cunha GM, Fowler KJ, Abushamat F, Sirlin CB, Kono Y. Imaging diagnosis of hepatocellular carcinoma. hepatocellular carcinoma: moving into the 21st century. Clin Liver Dis. 2020;24(4):623-636. doi:10.1016/j.cld.2020.07.002

32. Mitchell DG, Bruix J, Sherman M, Sirlin CB. LI-RADS (Liver Imaging Reporting and Data System): summary, discussion, and consensus of the LI-RADS Management Working Group and future directions. Hepatology. 2015;61(3):1056-1065. doi:10.1002/ hep. 27304

33. Kamath A, Roudenko A, Hecht E, et al. CT/MR LI-RADS 2018: clinical implications and management recommendations. Abdom Radiol. 2019;44(4):1306-1322. doi:10.1007/s00261-018-1868-6

34. Chaudhry M, McGinty KA, Mervak B, et al. The LI-RADS version 2018 MRI treatment response algorithm: evaluation of ablated hepatocellular carcinoma. Radiology. 2020;294(2):320-326. doi:10.1148/ radiol.2019191581

35. Ormiston WE, Yarmohammadi H, Lobaugh S, et al. Post-treatment CT LI-RADS categories: predictors of overall survival in hepatocellular carcinoma post bland transarterial embolization. Abdom Radiol. 2020;24:1.

36. King MJ, Tong A, Dane B, Huang C, Zhan C, Shanbhogue K. Response assessment of hepatocellular carcinoma treated with yttrium-90 radioembolization: inter-reader variability, comparison with 3D quantitative approach, and role in the prediction of clinical outcomes. Eur $J$ Radiol. 2020;133:109351. doi:10.1016/j. ejrad.2020.109351

37. Mendiratta-Lala M, Gu E, Owen D, et al. Imaging findings within the first 12 months of hepatocellular carcinoma treated with stereotactic body radiation therapy. Int J Radiat Oncol Biol Phys. 2018;102 (4):1063-1069. doi:10.1016/j.ijrobp.2017.08.022

38. Llovet JM, Lencioni R. mRECIST for HCC: performance and novel refinements. $J$ Hepatol. 2020;72(2):288-306. doi:10.1016/j. jhep.2019.09.026

39. Elsayes KM, Kielar AZ, Chernyak V, et al. LI-RADS: a conceptual and historical review from its beginning to its recent integration into AASLD clinical practice guidance. $J$ Hepatocell Carcinoma. 2019;6:49-69. doi:10.2147/JHC.S186239
40. Singal AG, Pillai A, Tiro J, Klenerman P. Early detection, curative treatment, and survival rates for hepatocellular carcinoma surveillance in patients with cirrhosis: a meta-analysis. PLoS Med. 2014;11(4):e1001624. doi:10.1371/journal.pmed.1001624

41. Morgan TA, Maturen KE, Dahiya N, Sun MRM, Kamaya A. US LI-RADS: ultrasound liver imaging reporting and data system for screening and surveillance of hepatocellular carcinoma. Abdom Radiol. 2018;43(1):41-55. doi:10.1007/s00261-017-1317-y

42. Hanna RF, Miloushev VZ, Tang A, et al. Comparative 13-year meta-analysis of the sensitivity and positive predictive value of ultrasound, CT, and MRI for detecting hepatocellular carcinoma. Abdoml Radiol. 2016;41(1):71-90. doi:10.1007/s00261-015-0592-8

43. An JY, Peña MA, Cunha GM, et al. Abbreviated MRI for hepatocellular carcinoma screening and surveillance. RadioGraphics. 2020;40 (7):1916-1931. doi:10.1148/rg.2020200104

44. Brunsing RL, Fowler KJ, Yokoo T, Cunha GM, Sirlin CB, Marks RM. Alternative approach of hepatocellular carcinoma surveillance: abbreviated MRI. Hepatoma Res. 2020;6. doi:10.20517/2394-5079.2020.50

45. Zaki IH, Shropshire E, Zhang S, et al. How frequently does hepatocellular carcinoma develop in at-risk patients with a negative liver MRI examination with intravenous gadobenate dimeglumine? Abdoml Radiol. 2020;19:1.

46. Rhee H, Kim H, Park YN. Clinico-radio-pathological and molecular features of hepatocellular carcinomas with keratin 19 expression. Liver Cancer. 2020;1-9. doi:10.1159/000505370

47. Chen J, Wu Z, Xia C, et al. Noninvasive prediction of HCC with progenitor phenotype based on gadoxetic acid-enhanced MRI. Eur Radiol. 2020;30(2):1232-1242. doi:10.1007/s00330-019-06414-2

48. Choi SH, Lee SS, Park SH, et al. LI-RADS classification and prognosis of primary liver cancers at gadoxetic acid-enhanced MRI. Radiology. 2019;290(2):388-397. doi:10.1148/radiol.2018181290

49. Choi JY, Kim MJ, Park YN, et al. Gadoxetate disodium-enhanced hepatobiliary phase MRI of hepatocellular carcinoma: correlation with histological characteristics. Am J Roentgenol. 2011;197 (2):399-405. doi:10.2214/AJR.10.5439

50. Kim JY, Kim MJ, Kim KA, Jeong HT, Park YN. Hyperintense HCC on hepatobiliary phase images of gadoxetic acid-enhanced MRI: correlation with clinical and pathological features. Eur J Radiol. 2012;81(12):3877-3882. doi:10.1016/j.ejrad.2012.07.021

51. Wei H, Jiang H, Liu X, et al. Can LI-RADS imaging features at gadoxetic acid-enhanced MRI predict aggressive features on pathology of single hepatocellular carcinoma? Eur $J$ Radiol. 2020;132:109312. doi:10.1016/j.ejrad.2020.109312

52. Choi SY, Kim SH, Park CK, et al. Imaging features of gadoxetic acidenhanced and diffusion-weighted MR imaging for identifying cytokeratin 19-positive hepatocellular carcinoma: a Retrospective Observational Study. Radiology. 2018;286(3):897-908. doi:10.1148/radiol.2017162846

53. Wei H, Jiang H, Zheng T, et al. LI-RADS category 5 hepatocellular carcinoma: preoperative gadoxetic acid-enhanced MRI for early recurrence risk stratification after curative resection. Eur Radiol. 2021;31(4):2289-302

54. Rhee H, An C, Kim HY, Yoo JE, Park YN, Kim MJ. Hepatocellular carcinoma with irregular rim-like arterial phase hyperenhancement: more aggressive pathologic features. Liver Cancer. 2019;8(1):24-40. doi: $10.1159 / 000488540$

55. Jeon SK, Joo I, Lee DH, et al. Combined hepatocellular cholangiocarcinoma: LI-RADS v2017 categorisation for differential diagnosis and prognostication on gadoxetic acid-enhanced MR imaging. Eur Radiol. 2019;29(1):373-382. doi:10.1007/s00330-018-5605-x

56. Min JH, Kim SH, Hwang JA, et al. Prognostic value of LI-RADS category on gadoxetic acid-enhanced MRI and 18F-FDG PET-CT in patients with primary liver carcinomas. Eur Radiol. 2020;1-2.

57. Kahn JCE, Heilbrun ME, Applegate KE. From guidelines to practice: how reporting templates promote the use of radiology practice guidelines. J Am Coll Radiol. 2013;10(4):268-273. doi:10.1016/j. jacr.2012.09.025 
58. Available from: https://www.RadLex.org. Accessed April 23, 2021.

59. Available from: https://www.radelement.org. Accessed April 23, 2021.

60. Kramer H, Pickhardt PJ, Kliewer MA, et al. Accuracy of liver fat quantification with advanced CT, MRI, and ultrasound techniques: prospective comparison with MR spectroscopy. Am J Roentgenol. 2017;208(1):92-100. doi:10.2214/AJR.16.16565

61. Ajmera VH, Liu A, Singh S, et al. Clinical utility of an increase in magnetic resonance elastography in predicting fibrosis progression in nonalcoholic fatty liver disease. Hepatology. 2020;71(3):849-860. doi:10.1002/hep.30974

62. Kurosaki M, Hosokawa T, Matsunaga K, et al. Hepatic steatosis in chronic hepatitis $\mathrm{C}$ is a significant risk factor for developing hepatocellular carcinoma independent of age, sex, obesity, fibrosis stage and response to interferon therapy. Hepatol Res. 2010;40(9):870-877. doi:10.1111/j.1872-034X.2010.00692.x

63. Ichikawa S, Motosugi U, Enomoto N, Onishi H. Magnetic resonance elastography can predict development of hepatocellular carcinoma with longitudinally acquired two-point data. Eur Radiol. 2019;29 (2):1013-1021. doi:10.1007/s00330-018-5640-7

64. Ajmera V, Park CC, Caussy C, et al. Magnetic resonance imaging proton density fat fraction associates with progression of fibrosis in patients with nonalcoholic fatty liver disease. Gastroenterology. 2018;155(2):307-310. doi:10.1053/j.gastro.2018.04.014

65. Liu X, Faes L, Kale AU, et al. A comparison of deep learning performance against health-care professionals in detecting diseases from medical imaging: a systematic review and meta-analysis. Lancet Digit Health. 2019;1(6):e271-e297. doi:10.1016/S2589-7500(19) 30123-2

66. Yamashita R, Mittendorf A, Zhu Z, et al. Deep convolutional neural network applied to the liver imaging reporting and data system (LI-RADS) version 2014 category classification: a pilot study. Abdom Radiol. 2020;45(1):24-35. doi:10.1007/s00261-019-02306-7
67. Peng J, Kang S, Ning Z, et al. Residual convolutional neural network for predicting response of transarterial chemoembolization in hepatocellular carcinoma from CT imaging. Eur Radiol. 2020;30 (1):413-424. doi:10.1007/s00330-019-06318-1

68. Hamm CA, Wang CJ, Savic LJ, et al. Deep learning for liver tumor diagnosis part I: development of a convolutional neural network classifier for multi-phasic MRI. Eur Radiol. 2019;29(7):3338-3347. doi:10.1007/s00330-019-06205-9

69. Byun J, Choi SH, Byun JH, et al. Comparison of the diagnostic performance of imaging criteria for $\mathrm{HCCs} \leq 3.0 \mathrm{~cm}$ on gadoxetate disodium-enhanced MRI. Hepatol Int. 2020;14(4):534-543. doi:10.1007/s12072-020-10040-2

70. Ren AH, Zhao PF, Yang DW, Du JB, Wang ZC, Yang ZH. Diagnostic performance of MR for hepatocellular carcinoma based on LI-RADS v2018, compared with v2017. J Magn Reson Imaging. 2019;50 (3):746-755. doi:10.1002/jmri.26640

71. An $\mathrm{C}$, Lee $\mathrm{CH}$, Byun $\mathrm{JH}$, et al. Intraindividual comparison between gadoxetate-enhanced magnetic resonance imaging and dynamic computed tomography for characterizing focal hepatic lesions: a multicenter, multireader study. Korean J Radiol. 2019;20 (12):1616. doi:10.3348/kjr.2019.0363

72. Park S, Joo I, Lee DH, et al. Diagnostic performance of LI-RADS treatment response algorithm for hepatocellular carcinoma: adding ancillary features to MRI compared with enhancement patterns at CT and MRI. Radiology. 2020;296(3):554-561. doi:10.1148/radiol.20 20192797

73. Youn SY, Kim DH, Choi SH, et al. Diagnostic performance of liver imaging reporting and data system treatment response algorithm: a systematic review and meta-analysis. Eur Radiol. 2021:1-9. doi:10.1007/s00330-020-07108-w
Journal of Hepatocellular Carcinoma

\section{Publish your work in this journal}

The Journal of Hepatocellular Carcinoma is an international, peerreviewed, open access journal that offers a platform for the dissemination and study of clinical, translational and basic research findings in this rapidly developing field. Development in areas including, but not limited to, epidemiology, vaccination, hepatitis therapy, pathology and molecular tumor classification and prognostication are all considered for publication. The manuscript management system is completely online and includes a very quick and fair peer-review system, which is all easy to use. Visit http://www.dovepress.com/ testimonials.php to read real quotes from published authors. 